\title{
Physical Experiment Research on Dielectric Properties of Hydrate-bearing Sediment in Sandstone Reservoir
}

\author{
Bing-rui Du, ${ }^{1,2,{ }^{*}}$ Da-wei Bai, ${ }^{1,2}$ Peng-hui Zhang, ${ }^{1,2}$ Peng Guo, ${ }^{1,2}$ Qiang Zhang, ${ }^{1,2}$ \\ ${ }^{1}$ Institute of Geophysical and Geochemical Exploration, CAGS, Langfang 065000, P.R. China; \\ ${ }^{2}$ National Research Centre of Geoexploration Technology, Langfang 065000, P.R. China;
}

\begin{abstract}
Dielectric constants can be used to detect hydrates in permafrost regions. Therefore, this study investigated the relationships between the dielectric constant characteristics of sandstone reservoir hydrate and the hydrate saturation degree through physical simulation experiments, as well as the granularity of the surrounding rock. Methane and tetrahydrofuran (THF) hydrates with quartz sands were prepared, and their dielectric constants were analyzed. With different granularities of quartz sands, the dielectric constants of two different methane hydrate sediments decreased with increasing saturation degrees. At a given saturation degree, the dielectric constant of methane hydrate sediments with small granularity was larger than that with medium granularity, a result attributed to the unreacted water in the larger pores of the latter. In addition, the dielectric constant of methane hydrate sediments was larger than that of THF hydrates, which was also attributed to gas-phase factors and the presence of unreacted water. At a given granularity and saturation, the dielectric constants of both the THF and methane hydrates decreased with increasing saturation degrees. We conclude that at low temperature and under normal pressure, THF hydrates cannot be used as a substitute for methane hydrates in laboratory experiments investigating geophysical phenomena.
\end{abstract}

\section{Introduction}

As a potentially vast source of energy, gas hydrate reserves have drawn increasing attention around the world. After years of research on the Qinghai-Tibet Plateau, Chinese geologists believe that the permafrost regions there possess the temperature and pressure conditions required to form gas hydrate, and a large amount of natural gas hydrate may exist in such regions ${ }^{[1]}$. In 2008, physical samples of natural gas hydrate were successfully drilled in the Muli district, Qilian Mountains in Qinghai Province. Hence, China became the first country to find natural gas hydrates in the midlatitude alpine permafrost region ${ }^{[2]}$.

Electromagnetic probe has been an effective method for the exploration and development of natural gas hydrates in permafrost regions. Chinese and international researchers have used it to conduct a number of related studies. Many geophysical and electromagnetic logging technologies were investigated for gas hydrate mineral deposits in the Mackenzie Delta in Canada and Alaska North Slope in the USA ${ }^{[3-4]}$. The Geological Survey of China has carried out geophysical exploration works and effectiveness experiments in the Muli area of Qilian Mountain, such as audio-frequency magnetotellurics, low frequency ground penetrating radar (GPR), surface nuclear magnetic resonance (NMR) method, and electromagnetic well logging technique ${ }^{[5]}$. However, after drilling, it is found that many identification marks are not completely related to natural gas hydrate deposits.
Therefore, the interaction between gas hydrates and electromagnetic waves and the electromagnetic response mechanism are not clear, and so many relevant interpretations are unconfirmed. In fact, gas hydrates can be obtained in only one-third of the drilling wells ${ }^{[6]}$.

Data from the natural gas hydrate well logging in Alaska North Slope was analyzed by Collett et al., and the energy potential of natural gas hydrates in the studied area was determined. These authors argued that well logging techniques are important for studying the in-situ physical properties of hydrates. Winters et al. studied the physical properties of sediments obtained from the stratum well logging of the Elbert gas hydrate in Alaska North Slope. A clear radar wave emission signal was found in the hydrate reservoir, while the reflected signal was characterized by "high frequency" and "strong amplitude" which can be regarded as the electromagnetic identification mark of gas hydrate in permafrost regions. The dielectric constants of DK-8 well core samples in the Muli area were studied by $\mathrm{Li}$ et al ${ }^{[7]}$, who argued that in the process of natural gas hydrate formation, the dielectric constant of the system had a complex change rule and dispersion characteristics. The electromagnetic prospecting technology based on the dielectric constant can be used to quantitatively analyze the hydrate strata. However, it is difficult to use the same technology to quantify hydrate saturation. Traditional geophysical and lithophysical approaches can only characterize formations at the macro-scale or describe the surface morphology of rock samples, and few studies have

\footnotetext{
*Corresponding author: 125110371@qq.com
} 
examined the geophysical response characteristics of cores from the drilled area ${ }^{[8]}$.

In the Alaska North Slope, direct evidence of natural gas hydrates comes from studying the cores from two natural gas hydrate test wells ${ }^{[9-10]}$. Geophysical logging well drilled in the MacKenzie Delta shows that there is a significant difference in the dielectric constants between hydrate-bearing and non-hydrate-bearing formations in the same sedimentary formations ${ }^{[11]}$. The high-frequency electromagnetic wave-synchronous radiation X-ray source technology was used by foreign scholars to characterize and quantitatively analyze the internal structure of core samples. The micro-scale gap morphology of hydrate rocks was obtained, thereby characterizing the 3D spatial distribution of hydrates. Comprehensive geophysical well logging was conducted by Zhu et al ${ }^{[12]}$. by drilling hydrate-bearing formation segments. The results showed that the natural gas hydrate segments exhibited relatively high resistivity and high wave velocity, which are significantly different from the experimental data reported so far. Simultaneous experimental measurement of hydrate saturation, electromagnetic wave velocity, and dielectric constant in the Muli area has not been available. So, there is insufficient evidence to explain the direct correspondence between hydrate saturation and the dielectric properties in the permafrost region ${ }^{[13-14]}$.

This paper describes preliminary experimental work on the laboratory synthesis of models to simulate hydrate-bearing sediment from the drilled hydrate reservoirs, and in-situ measurement of their electromagnetic responses. The test samples were hard rock cores dominated by sandstone reservoirs, since few cores in the drilling area can be preserved from reservoirs in the Muli area because of the loose and fragile mudstone and shale. The main research objectives are: (1) to elucidate whether there are distinct dielectric properties of methane hydrate in sandstone; (2) to check whether artificially synthesized sandstone-methane hydrate has regular electromagnetic wave response characteristics, and, if yes, the influencing factors, and (3) to analyze the difference in electromagnetic responses from THF hydrate (a substitute of methane hydrate in laboratory) and methane hydrate itself.

\section{Materials and Methods}

\subsection{Experimental principle}

The dielectric constant $\varepsilon$, also known as the permittivity, characterizes the electrical properties of a dielectric or insulating material and represents the relative electrostatic energy storage of this material in an electric field. $\varepsilon$ is defined as the ratio of the capacitance of a capacitor filled with the given material to that of an identical capacitor in vacuum without the dielectric material.

Gas hydrates are difficult to obtain in situ, since they generally exist only at low temperature and high pressure, and are easily decomposed by disturbances in these conditions. Laboratory-based simulation of hydrate reservoirs in the frozen soils can provide abundant data closely related to the response characteristics of natural dense rock to electromagnetic waves, thereby guiding the field exploration work. Under low temperature and pressure conditions, we can model the sandstone hydrate by a mixture of sandstone particles in different sizes and artificially synthesized methane hydrate. Because the volume of methane hydrate produced by the reactor is fixed, hydrate sediment samples with different saturations are prepared by changing the volume of quartz sand in the mixture. High-frequency GPR is then applied to these samples to measure the different predetermined saturations and the corresponding dielectric constants. Since this experiment focuses on the rule of response, the saturation in the model (Sh) can be approximated as

$$
\frac{V_{h}}{V_{s} \times \Phi_{\mathrm{s}}} \cong S_{h}
$$

where $\mathrm{Vh}$ is the volume of synthesized hydrates, the volume of synthetic methane hydrate in the reactor is fixed at $1 \mathrm{~L}, \mathrm{Vs}$ is the volume of the quartz sand, and $\Phi \mathrm{s}$ is the porosity of quartz sand (measured to be $30 \%$ ).

\subsection{Experimental instrument}

Considering the test time, the stability of the transmitted signal, and the depth of the investigation (corresponding to the thickness of the model), the SIR-3000 highfrequency GPR system (GSSI Inc., USA) was used. The radar antenna was tuned to $1500 \mathrm{MHz}$, the scanning rate was set to be 192 lines per second, the transmitting power was $100 \mathrm{kHz}$, the gain was set manually at 3 nodes, and point measurements were performed with single-channel recording. The data were processed using the Reflexw software (Sandmeier Geophysical Research, Germany), and the dielectric constant of the model was obtained by averaging the waveforms after singlechannel processing.

\subsection{Procedure for measuring the dielectric constant over medium-grained sandstone methane hydrate}

\subsubsection{Preparation of the experimental samples}

Table 1. Constituents of medium-grained sandstone methane (CH4) hydrate sediments.

\begin{tabular}{|l|l|}
\hline Constituent & Source \\
\hline $\begin{array}{l}\text { Distilled } \\
\text { water (H2O) }\end{array}$ & Prepared in lab \\
\hline $\begin{array}{l}\text { Methane } \\
(\mathrm{CH} 4,>99.9 \%\end{array}$ & Haowen Gas \\
\hline
\end{tabular}




\begin{tabular}{|l|l|}
\hline ) & Factory, China \\
\hline $\begin{array}{l}\text { Quartz sand } \\
\text { (SiO2) }\end{array}$ & $\begin{array}{l}\text { Domestic } \\
\text { artificial } \\
\text { synthesis }\end{array}$ \\
\hline $\begin{array}{l}\text { Sodium } \\
\text { dodecyl } \\
\text { sulfate } \\
\text { (SDS, >99.7\% } \\
\text { ) }\end{array}$ & $\begin{array}{l}\text { TuanKai } \\
\text { Technology Co, China }\end{array}$ \\
\hline
\end{tabular}

In the laboratory test, the surrounding rock material was experimentally modeled as soil samples prepared using sub-spherical uniform quartz sands (35-50 mesh) with the same granular level as the sediment framework for the hydrate. The stacking density, porosity, average grain diameter, and dielectric constant of medium-grained sandstone were $2.82 \mathrm{~g} / \mathrm{cm}^{3}, 0.3,400 \mu \mathrm{m}$, and 3 , respectively; thus the sandstone is a low-loss medium with respect to electromagnetic waves. Before the test, quartz sands were placed in a $-10^{\circ} \mathrm{C}$ freezer for more than 48 hours. A thick-walled bubble chamber with a cap was used as the soil sample load-bearing container with a length, width, and height of 54, 38.5, and $25 \mathrm{~cm}$, respectively, and a wall thickness of $3 \mathrm{~cm}$.

The major components of the experimental setup were a reactor, thermostatic water bath, gas input and output system, data acquisition system (temperature and pressure), and auxiliary equipment. Figure 1 shows a schematic of the experimental setup for methane hydrate formation.

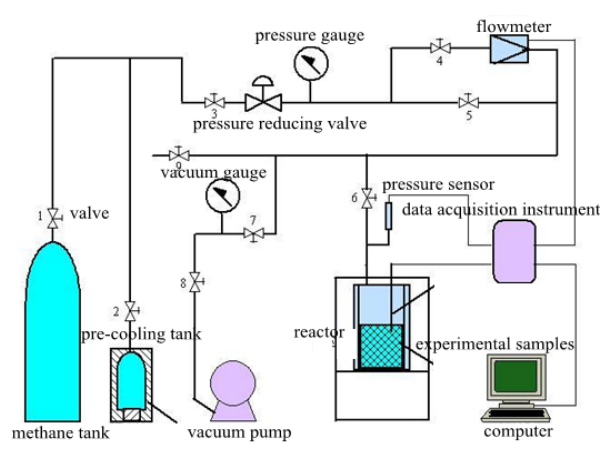

Figure 1 Experimental setup for methane hydrate formation

During the production of methane hydrate, the hydrate growth in the reaction kettle undergoes three processes: nucleation, an increase in the number of hydrate particles, and the accumulation of hydrate particles:

The number of hydrate particles rapidly increases, and visibility rapidly decreases;

The hydrate particles accumulate, the solution viscosity increases, and the height of the gas-liquid interface decreases; and

Hydrates form massive hydrate crystals associated with a certain amount of bubbles.
A volume of $1 \mathrm{~L}$ methane hydrate was produced in 48 hours by adding two parts per thousand of sodium dodecyl sulfate (SDS) as a catalytic agent at $6 \mathrm{MPa}$ and $273 \mathrm{~K}$. The methane hydrate sediment model was made in the laboratory by placing quartz sand in a lowtemperature chamber for more than 10 hours. A stirrer was used to break the hydrate on the inner wall of the reactor into particles or powder, which was then poured onto the quartz sands and mixed. Then, the saturation degree was adjusted using the volume ratio. In order to ensure the homogeneity of soil samples, the hydrate powder was divided into five layers and tamped. The compaction process used a steel plate of the same size as the top surface of the model. After each layer of hydrate and sandstone was stirred, it was squeezed from the top. The layering and squeezing steps were repeated 5 times to reduce the gaps between sandstone particles in each layer, thereby creating a uniform degree of compaction for the different models.

The interior of the sample may contain ice-like methane hydrate particles of different sizes, which are interspersed and in contact with the quartz sands. The presence of internal cracking in the mixed soil samples indicated that the decomposition of methane hydrate still occurred at low temperature and pressure, and therefore the soil samples had to be tested quickly.

\subsubsection{GPR test}

Based on the two-way travel time of the radar wave propagation, the passage time $\Delta \mathrm{t}$ of the radar wave in the THF model was calculated based on the time from the top to the bottom boundary, and the propagation velocity VTHF in the hydrate model can be calculated from the known model thickness. The dielectric constant of hydrate sediments can then be calculated as

$$
\varepsilon=\mathrm{V}_{\mathrm{K}}^{2} / V_{T H F}^{2}
$$

where VK is the propagation velocity of the radar wave in $\operatorname{air}(30 \mathrm{~cm} / \mathrm{ns})$. The experimental setup is shown in Figure 2.

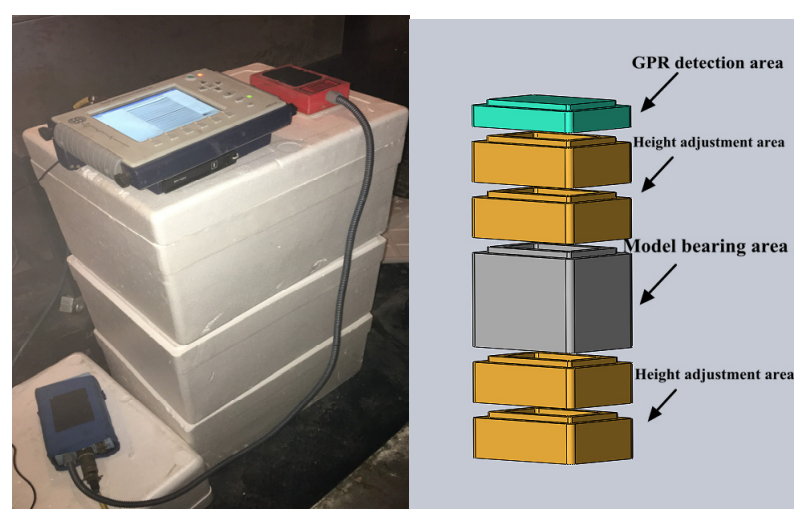

Figure 2 Experimental setup for obtaining dielectric constant of hydrate sediments 
Hydrate sediment models with different saturation degrees (5-50\%) were separately made. The methane hydrates were tested with the high-frequency GPR. Figures 3 show the sir-3000 radar test results for 30\% saturation to illustrate the single-channel signal extraction of the radar wave, respectively.

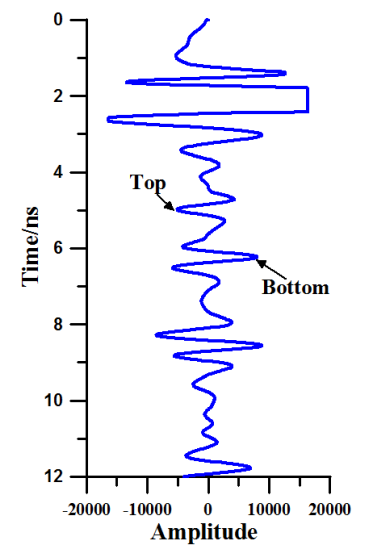

Figure 3 GPR single-channel signal extraction(30\% saturation)

The figure shows that the refraction and reflection are clearly impacted by differences in the dielectric constants encountered by the radar wave as it passes through the sample. According to the waveform profiles of the forward model, the radar wave reflection signals from the top and bottom boundaries of the methane hydrate are clear, and the waveform curves are regular in these areas. The signal from the top boundary represents the radar wave travelling from the air to the methane hydrate boundary, i.e., from an optically thinner medium to an optically denser medium. The reflection signal goes from zero in the beginning to a small positive peak and finally to a large negative peak in the reverse direction; the radar wave's travel time into the top boundary can be calculated according to the position of the negative peak.

\subsection{Procedure for testing the dielectric constant of micropsammite methane hydrate}

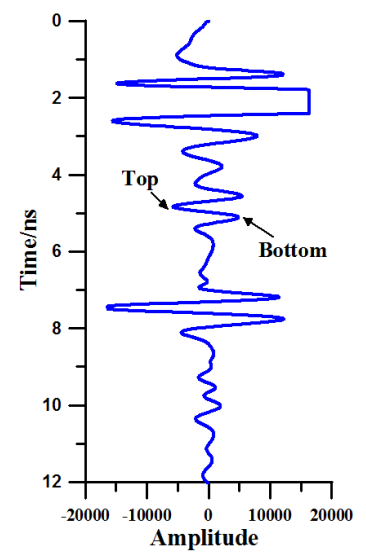

Figure 4 GPR single-channel signal extraction( micropsammite)
Figures 4 show the sir-3000 radar test results for $30 \%$ saturation of micropsammite methane hydrate to illustrate the single-channel signal extraction of the radar wave, respectively.

\subsection{Procedure for testing the dielectric constant over THF hydrate}

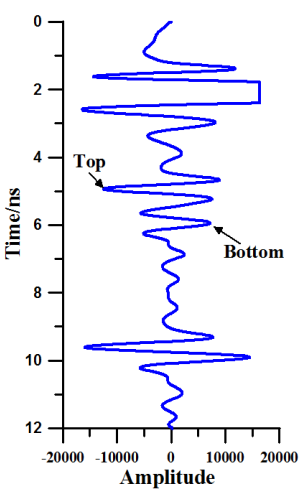

Figure 5 GPR single-channel signal extraction(THF)

Figures 5 show the sir-3000 radar test results for $30 \%$ saturation of THF hydrate to illustrate the single-channel signal extraction of the radar wave, respectively.

\section{Conclusions}

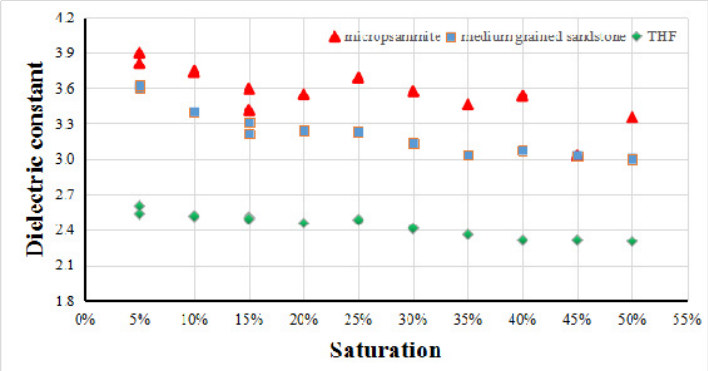

Figure 6 Comparison of the dielectric constants of three hydrate-bearing sediments at the same saturation degrees

A series of hydrate-bearing sediment models were prepared in the laboratory, and the electromagnetic properties of methane hydrate sediments and THF hydrate sediments were measured using high-frequency GPR. Various factors influencing their dielectric constants and the general trends for the dielectric constants were obtained. The main conclusions are summarized as follows.

1.A change of the microstructure in the model has a very significant impact on its electromagnetic response characteristics and dielectric properties. For both the methane and THF hydrates, the dielectric constants of the sediments decreased with increasing saturation degrees within a certain range, as the propagation velocity of the radar wave through the samples increased with increased hydrate volume. In addition, the physical properties of the internal model structure change with the degree of saturation: quartz sand ceases to be the main control factor and is replaced by hydrate content. 
2.The dielectric constant is very sensitive to changes in the filling of the pores. At a given saturation degree, the dielectric constant of the methane hydrate sediments with small rock granules was larger than that of the THF hydrate. However, the difference in their dielectric constants was small, and was mainly caused by unreacted water and non-exhausted air in the sample's pores.

3. When the methane hydrate saturation in the sandstone reservoir is greater than $15 \%$, the hydrate reservoir and surrounding rock have more detailed electromagnetic and dielectric properties. This condition is well suited for verification of ground penetrating radar and dielectric logging measurements.

4.The different hydrate sediment models and their dielectric constant characteristics revealed in this study provide a reference for in-situ measurements using geophysical, geochemical, dielectric logging, and electromagnetic wave logging approaches, etc.

Acknowledgments: This research was supported by the Chinese Academy of Geological Sciences basic research projects (AS2019P01). The authors especially thank Professor Fang Hui and Professor Xiao Du for their support of this experiment.

Author information: Bing-rui Du, male ,born in 1984, is currently engaged in the research and application of natural gas hydrate exploration technology at the Institute of Geophysical and Geochemical Exploration of the Chinese Academy of Geological Sciences.

\section{References}

1. Zhong, S.; Xiong, J.; Meng, Y.; et al. Strategic deliberation on unconventional energy source in China of 21st Century. Drill. Produc. Technol. 2005, 28(5), 93-98.

2. Zhu, Y.; Zhang, Y.; Wen, H.; et al. Gas hydrate in the Qilian mountain permafrost, Qinghai, northwest China. Acta Geolog. Sin. 2009, 83(11), 1762-1771.

3. Sun, Y.; Goldberg, D.; et al. High-resolution welllog derived dielectric properties of gas-hydratebearing sediments, Mount Elbert Gas Hydrate Stratigraphic Test Well, Alaska North Slope. Marin. Petrol. Geol. 2011, 28, 450-459.

4. Riedel, M.; Brent, T. A.; et al. Evidence for gas hydrate occurrences in the Canadian Arctic Beaufort Sea within permafrost-associated shelf and deepwater marine environments. Marin. Petrol. Geol. 2017, 81, 66-78.

5. Fang, H.; Sun, Z. Main achievements of gas hydrate exploration technology in permafrost regions of China. Geophys. Geochem. Explor. 2017, 6, 991997.

6. Lu, Z.; Zhu, Y.; et al. Basic geological characteristics of gas hydrates in Qilian Mountain permafrost area, Qinghai province. Miner. Depos. 2010, 1(29), 182-191.
7. Zhao, G.; Chen, X.; et al. Advanced geoelectromagnetic methods in China. Progr. Geophys. 2007, 4(22), 1171-1180.

8. Tong, M.; Li, L.; et al. Estimation of pore size distribution and permeability of shaly sands from induced polarization time spectra. Chin. J. Geophys. 2005, 3(48), 710-716.

9. Sun, Z.; Liu, C. Experimental study on water content measurement in THF hydrate system with time domain reflectometry (TDR). Marine Geology Frontiers 2012, 28(5), 64-70.

10. Hu, G.; Ye, Y.; Diao, S.B.; et al. Research of time domain reflectometry in measuring water content of marine sediments. Geosci. 2010, 24(3), 622-626. DOI: 10.3969/j.issn.1000-8527.2010.03.029.

11. Du, B.; Bai, D. Preparation of laboratory hydrate physical model and research on the electromagnetic properties based on the ground penetrating radar. Comput. Tech. Geophys. Geochem. Explor. 2016, 38(4), 467-472.

12. Chen, B.; Xu, J.; et al. Hydrocarbon source for oil and gas indication associated with gas hydrate and its significance in the Qilian Mountain permafrost, Qinghai, Northwest China. Marin. Petrol. Geol. 2018, 89, 202-215.

13. Wang, P.; Zhu, Y.; et al. Gas hydrate in the Qilian Mountain permafrost and its distribution characteristics. Geol. Bull. Chin. 2011, 30(12), 1839-1850.

14. Chen, L.; Qin, R.; et al. Structural fracture characteristics of cores from gas-hydrate drillholes in Sanlutian of Muli coalfield, Qinghai. Geoscience 2015, 5(29), 1087-1095.

15. Li, C.; Liu, C.; et al. CT image characterization of pores and fissures in rock core from Juhugeng gas hydrate area in Qinghai. Geoscience 2015, 5(29), 1189-1193. 